\title{
Los hogares conyugales con doble proveeduría en Colombia. Construcción de una tipología de arreglos laborales con enfoque de género'
}

\author{
Conjugal Homes with Double Supply in Colombia. \\ Contributions for the Construction of a Typology of \\ Labor Arrangements with Gender Approach
}

Rosa Emilia Bermúdez-Rico ${ }^{2}$

Profesora e investigadora del Departamento de Ciencias Sociales de la Universidad del Valle, Cali, Colombia

rosa.bermudez@correounivalle.edu.co

ORCID: https://orcid.org/0000-0003-2500-6719

Paola Julieth Melo-Morales ${ }^{3}$

Asistente de investigación de la Universidad del Valle, Cali, Colombia

paola.melo@correounivalle.edu.co

ORCID: https://orcid.org/0000-0002-7246-4846

Recibido: 06-02-2018

Aprobado: 20-03-2019

1 "Caracterización de las clases medias y movilidad social en espacios urbanos, un estudio comparado", financiado por la Convocatoria interna de la Universidad del Valle y la Universidad de Los Andes en el año 2015, y del proyecto "Y si ambos trabajan, qué sucede en el hogar: división sexual del trabajo en hogares con doble proveedor en Cali”, finalizado en el año 2017.

Doctora en Estudios de Población.

3 Socióloga. 


\section{Resumen}

El propósito de este artículo es establecer una clasificación de los arreglos laborales de los hogares conyugales con doble proveeduría en Colombia, considerando la participación en el mercado laboral diferenciada por sexo de los integrantes de la pareja conyugal. Este análisis contribuye a comprender el significado que adquieren las relaciones entre trabajo y género en este tipo de hogares en las sociedades contemporáneas. Con este propósito se establece la categoría analítica "hogar conyugal según arreglo laboral de la pareja" y se procesa la información suministrada por la Encuesta Nacional del Uso del Tiempo (ENUT 2012-2013). Con este procesamiento se establece una tipología de los arreglos laborales que se identifica en los hogares colombianos. El análisis realizado resalta la alta participación que ha alcanzado este tipo de hogar en los contextos urbanos en el país y subraya el alto nivel educativo que presentan las parejas que lo configuran.

Palabras clave: división sexual del trabajo; hogares conyugales; doble proveeduría; género; participación laboral.

\section{Abstract}

The purpose of this article consists in establishing a classification labor arrangements of conjugal homes with double supply in Colombia, considering the participation in the market understanding the differences between sexs in a conjugal relationship. This analysis contributes to a better understanding of the meaning between "work" and "gender" in these kind of homes in contemporary societies. With that purpose in mind, we establish the analytical category "conjugal home according to labor arrangement", and all the information provided by the Nacional del Uso del Tiempo survey (ENUT 2012-2013) is processed. With this procedure, a typology of labor arrangements that we identify in Colombian homes is established. The analysis emphasizes the high participation levels that these type of homes have reached in urban contexs in the country and the high educational level of the couples that conform said homes.

Key words: Conjugal Homes, Double Supply, Gender, Labor Arrangements.

\section{¿Cómo citar este artículo? / How to quote this article?}

Bermúdez-Rico, R. E. y Melo-Morales, P. J. (2019). Los hogares conyugales con doble proveeduría en Colombia. Construcción de una tipología de arreglos laborales con enfoque de género. Sociedad y economía, (37), 33-49. https://doi.org/10.25100/sye. voi37.7820 
Los hogares conyugales con doble proveeduría en Colombia. Construcción de una tipología de arreglos laborales con enfoque de género

\section{Introducción}

A partir de la inserción de la mujer en el mercado laboral se han presentado transformaciones en la configuración de los hogares y las familias en América Latina, en las últimas décadas (Arriagada y Aranda, 2004; Quilodrán, 2011; Rendón, 2004). Transformaciones asociadas a la postergación del matrimonio, la reducción del tamaño de los hogares, el descenso de la tasa de fecundidad, la ampliación del nivel educativo, la emergencia de nuevas estructuras familiares y la consolidación de valores que resaltan la autonomía y el desarrollo personal ${ }^{4}$ (Arriagada, 2004; Ariza y de Oliveira, 2001; Therborn, 2007; Ministerio de Salud y Protección Social y Profamilia, 2016).

Las transformaciones sociodemográficas señaladas están fuertemente articuladas con cambios en la organización económica y la reproducción cotidiana de los hogares, así como con los roles económicos que hombres y mujeres desempeñan en la familia. En este marco de referencia, se puede afirmar que las transformaciones ocurridas en el mercado laboral están estrechamente relacionadas con las pautas de sostenimiento económico, las dinámicas familiares y con las relaciones de género. De manera que, en las últimas décadas, hemos transitado desde el modelo hegemónico de representación de la familia (con un hombre proveedor económico, bajo la figura de esposo-padre, y una mujer que realiza el trabajo doméstico y de cuidado en el hogar, bajo la figura de esposa-madre considerada inactiva económicamente), hacia la configuración de diversos modelos de organización familiar que han reconocido e incorporado la actividad económica de las mujeres y han visibilizado nuevas condiciones de poder en el hogar (Wainerman, 2007). Esta transición en la configuración de los hogares, ha convocado a las ciencias sociales a una nueva y

4 De manera semejante que en otros países de América Latina, Colombia ha presentado una reducción de la tasa de fecundidad en los últimos años, pasando de 3,0 en 1990-1995, a 2,55 en 2000-2005, hasta 2,36 en 20102015. Así mismo, ha presentado desde mediados del siglo pasado un aumento sostenido en la inserción de las mujeres al mercado laboral (Ullmann, Maldonado, y Nieves, 2014), en tanto la Tasa Global de Participación (TGP) femenina se estimó en 19,0\% para el año 1950, mientras que en 1995 se estimó una tasa de 49,1\%, y para el año 2000 se estimó en 58,6\% (Martínez, 2013). amplia reflexión en torno a la categoría analítica de división sexual del trabajo; ha actualizado la discusión de la interrelación entre ámbitos públicos y privados, y ha puesto de presente el debate en torno a la incidencia de estas dinámicas de cambio en las construcciones identitarias de género (Ariza y de Oliveira, 2009; Arriagada y Aranda, 2004; Jelin, 2007).

En este marco general se puede señalar que -si bien el modelo de único proveedor económico, constituido como familia nuclear biparental con hijos/as, fue predominante en el siglo XX- en las décadas recientes se observa una disminución de este tipo de hogar y el aumento de otros tipos de hogares nucleares. Tal como señalan Arriagada y Aranda (2004), de 1990 a 2002, el principal cambio entre los hogares nucleares ha sido el aumento de los hogares biparentales donde ambos cónyuges están activos económicamente: tanto con hijos como sin hijos, pasaron de representar $27 \%$ a $33 \%$, y de $4 \%$ a $5 \% 5$, respectivamente, del total de hogares nucleares, en las zonas urbanas, de dieciocho países de América Latina ${ }^{6}$. Colombia se encuentra inscrita en el marco general de esta tendencia: tal como señalan los datos, los hogares nucleares con ambos cónyuges activos, con hijos y sin hijos en el país, entre 1991 y 2002, pasaron de $28,4 \%$ a $32,8 \%$, y de $3,4 \%$ a $5,2 \%$, respectivamente 7 .

5 Otros de los hogares nucleares, que han presentado una disminución de su participación entre 1990 a 2002, corresponde a los hogares biparentales con hijos con un hombre que trabaja (de $47 \%$ a $36 \%$ ). En contraste, han incrementado los hogares monoparentales: con jefa mujer que trabaja de forma remunerada (de $8 \%$ a $10 \%$ ), con jefa mujer que no trabaja (de $5 \%$ a $6 \%$ ), y con jefe hombre que trabaja (de $2 \%$ a $3 \%$ ) (Arriagada y Aranda, 2004).

6 La información procesada corresponde a los siguientes países: Argentina, Bolivia, Brasil, Chile, Colombia, Costa Rica, Ecuador, El Salvador, Honduras, México, Nicaragua, Panamá, Paraguay, Perú, República Dominicana, Uruguay, Venezuela. Las fuentes utilizadas son representativas a nivel nacional (Arriagada y Aranda, 2004).

7 De manera semejante para el caso de Latinoamérica, los hogares con un proveedor en Colombia con hijos, también presentaron un descenso de su participación: de 47,0\% en 1991, a 35,1\% en 2002. En este mismo periodo, los hogares monoparentales incrementaron su participación: los hogares monoparentales con jefatura femenina, con mujer que trabaja, pasaron de $8,9 \%$ a $1,4 \%$, y con mujer que no trabaja, pasaron de $6,0 \%$ a $6,7 \%$. Así mismo, los hogares monoparentales con jefatura masculina pasaron de 1,6\% a 2,5\% (Arriagada y Aranda, 2004). 
En suma, las investigaciones realizadas en los países de la región, por un lado, convergen en señalar la presencia de importantes procesos de transformación en las estructuras familiares que han conllevado a diversas configuraciones en los hogares. Por otro lado, coincide en enfatizar la persistencia en los patrones de la división sexual del trabajo que colocan en desventaja a las mujeres, asignándoles el rol principal en el trabajo doméstico y de cuidado, incluso en aquellos hogares con doble proveeduría. En este contexto, han indicado que se tiene un avance parcial en la igualdad de oportunidades para las mujeres y los hombres tanto en la esfera privada como en la pública (Sánchez, 2014; Sosa, 2015; Wainerman y Heredia, 2000).

En el contexto de las transformaciones señaladas, este artículo presenta el análisis de los hogares conformados por parejas conyugales en Colombia y, en particular, presenta una estimación y clasificación de los hogares con doble proveeduría en el país. Hasta el momento, para Colombia no se ha realizado una estimación de los hogares conyugales que nos permita aproximarnos a su magnitud, así como tampoco se han identificado sus diversas configuraciones actuales en el contexto del país. En esta misma línea, no disponemos de una caracterización que nos permita establecer los arreglos laborales tanto para aquellos hogares de un solo proveedor como con doble proveeduría. Este análisis permitirá aportar un mayor conocimiento sobre los patrones distintivos de las relaciones entre trabajo, familia y género en estos tipos de hogares.

El procesamiento de la información se fundamenta en los datos suministrados por la Encuesta Nacional del Uso del Tiempo (ENUT, 2012-2013). La ENUT es una encuesta aplicada con el fin de generar información sobre el tiempo dedicado, por la población de 10 años y más, a actividades de trabajo remunerado, no remunerado y personales. Esta encuesta tiene cubrimiento nacional y se realiza bajo un diseño muestral probabilístico, estratificado, de conglomerados y polietápico; es apli- cada por el Departamento Administrativo Nacional de Estadística en Colombia -DANE- ${ }^{8}$.

Para el desarrollo del análisis propuesto se consideran los hogares conformados por parejas conyugales, a partir de la selección de casos, utilizando la siguiente definición operativa: población que perteneciendo a un mismo hogar afirma tener un parentesco correspondiente a jefe o cónyuge, es decir, conforman una pareja conyugal. Para la delimitación de los hogares conyugales con doble proveedores se ha establecido la participación de cada uno de los cónyuges en el mercado laboral y se ha incluido parejas con un estado civil casado o en unión libre.

En tal sentido, el incremento de los hogares con doble proveedores en las últimas décadas, su relevancia en la discusión en torno a las implicaciones de la inserción de las mujeres en el mercado laboral -así como de las transformaciones en los roles e identidad de género, y la organización y dinámicas de las familias contemporáneas-, conllevan a subrayar la importancia de realizar un análisis de los hogares conformados por parejas conyugales de acuerdo a la participación laboral, tanto de hombres como mujeres, así como del estatus de proveedor de cada integrante en el contexto colombiano.

El artículo se estructura de la siguiente manera: en primer lugar, se presenta una tipología de los hogares conformados por parejas conyugales a partir de la caracterización de los arreglos laborales identificados en estas parejas; considerando la articulación de variables estratégicas que han sido señaladas en los debates en torno a la configuración de los hogares conyugales de doble proveeduría. En segunda instancia, se presenta un análisis de la clasificación y las estimaciones para cada uno de los tipos de hogares de parejas conyugales en Colombia, y se determina en particu-

8 La muestra consultada está conformada por 148.492 personas y 43.500 hogares total nacional. Para el análisis de los hogares conyugales en las zonas urbanas, se clasificaron 19.264 hogares. 
Los hogares conyugales con doble proveeduría en Colombia. Construcción de una tipología de arreglos laborales con enfoque de género

lar el nivel de participación de los hogares de doble proveeduría en el país. En un tercer apartado, se presenta una discusión sobre el lugar que ocupa y el significado que adquieren las parejas con doble proveeduría en la estructura general de los hogares conyugales en Colombia, y se establece una distribución de las parejas con doble proveeduría según el nivel educativo; además se analiza en detalle esta distribución en los hogares conyugales conformados por hombres y mujeres pertenecientes a las cohortes consideradas en el rango de edad entre 25 y 40 años. Para cerrar, se presentan algunas reflexiones generales.

\section{Los hogares con pareja conyugal según número de proveedores, participación laboral y sexo: una propuesta de tipos analíticos}

La clasificación de los hogares con pareja conyugal que presentamos a continuación se fundamenta en un enfoque teórico que considera la importancia del concepto de división sexual del trabajo y reconoce una clara diferenciación de los roles de género en la organización de la vida familiar. Esta clasificación responde al objetivo general de discutir distintas formas de organización familiar, considerando la participación laboral de los hombres y de las mujeres que conforman las parejas conyugales.

En este contexto, se subraya que la división sexual del trabajo predominante en las sociedades contemporáneas ha privilegiado la representación de un hombre proveedor del sustento económico del hogar y de una mujer ama de casa proveedora del trabajo doméstico y de cuidado. Por ello, resulta relevante desarrollar un análisis de sexo-género que permita hacer explícitos aspectos particulares de los arreglos conyugales establecidos por cada tipo de hogar para la participación en el mercado laboral. En tal dirección, se retoma la categoría de análisis de género en tanto aporta a la explicación de las desigualdades entre varones y mujeres, así como a la comprensión de lo que cada sociedad establece cultural e históricamente como rasgos socialmente legí- timos para definir los roles femeninos y masculinos (Rodríguez, 2015). De igual forma, siguiendo a West y Zimmerman (1999) se resalta que "el género se hace" socialmente en la interacción recurrente con los otros y alcanza una expresión institucional. En el análisis realizado, se parte de reconocer la división sexual del trabajo como una forma institucionalizada de hacer género, en tanto el género se constituye en su principio articulador y organizador; es en este sentido que se afirma que la división sexual del trabajo es un proceso generizado.

Por otro lado, los análisis de las transformaciones de los mercados laborales en los países de América Latina han señalado su importancia en el impacto en los roles económicos y de género en la esfera familiar. Este impacto ha estado estrechamente asociado con el aumento sostenido de la inserción laboral femenina y con un incremento relativo del desempleo masculino. De allí que resulte relevante distinguir la situación de cada cónyuge en el mercado laboral, referente a la posición de: ocupado, desocupado, e inactivo económicamente. Bajo esta perspectiva conceptual se retoma la propuesta de categorías analíticas sugerida por Wainerman y Heredia (2000).

La clasificación de los hogares se establece bajo la articulación de los siguientes criterios: el sexo de los cónyuges y la categoría diferenciada en la participación laboral que cada uno de ellos tiene. Bajo este esquema de clasificación se obtienen tres grandes tipos de arreglos laborales para los hogares conyugales: parejas con un único proveedor económico (Tipo I), parejas con dos proveedores económicos (Tipo II) y parejas inactivas laboralmente, sin ningún proveedor (Tipo III). A su vez, en estos tres grandes tipos se pueden diferenciar distintas combinaciones o arreglos del estatus laboral de cada uno de los cónyuges (Tabla 1).

9 Para los fines analíticos en esta clasificación se ha considerado que la participación en el mercado laboral tanto en la condición de ocupado como desocupado, es equivalente a la condición de proveedor económico en tanto los datos disponibles no permiten hacer una distinción al respecto y se ha considerado que disponer de ingresos (por remuneración al trabajo, renta o pensión), por parte de cualquiera de los cónyuges, automáticamente lo constituye en proveedor económico en el hogar. 


\begin{tabular}{|c|c|}
\hline $\begin{array}{l}\text { Tipos de arreglo laboral de } \\
\text { la pareja conyugal }\end{array}$ & Definición \\
\hline $\begin{array}{l}\text { TIPO I: } \\
\text { Un proveedor económico }\end{array}$ & $\begin{array}{l}\text { Se trata de hogares que cuentan con un cónyuge que participa en el mercado laboral }{ }^{\mathrm{a}} \text {, } \\
\text { ya sea como ocupado o desocupado }{ }^{\mathrm{b}} \text { y otro que se encuentra inactivo económica- } \\
\text { mente. En este tipo de hogar se consideran las situaciones diferenciadas del proveedor } \\
\text { económico, primero, de acuerdo al sexo y, segundo, por la participación laboral. }\end{array}$ \\
\hline $\begin{array}{l}\text { Tipo IA: } \\
\text { Un proveedor hombre }\end{array}$ & $\begin{array}{l}\text { Agrupa a aquellos cónyuges caracterizados por un hombre que participa en el mercado } \\
\text { laboral y una mujer que es inactiva económicamente. }\end{array}$ \\
\hline $\begin{array}{l}\text { Hombre ocupado, mujer } \\
\quad \text { inactiva }\end{array}$ & $\begin{array}{l}\text { Se refiere a aquellas parejas conyugales conformadas por un hombre ocupado y una } \\
\text { mujer inactiva económicamente. }\end{array}$ \\
\hline $\begin{array}{l}\text { Hombre desocupado, mujer } \\
\text { inactiva }\end{array}$ & $\begin{array}{l}\text { Hace referencia a las parejas conyugales que cuentan con un hombre que participa en } \\
\text { el mercado laboral bajo la condición de desocupado o en busca de trabajo remunerado } \\
\text { y una mujer inactiva económicamente. }\end{array}$ \\
\hline $\begin{array}{l}\text { Tipo IB: } \\
\text { Una proveedora mujer }\end{array}$ & $\begin{array}{l}\text { Abarca los hogares conyugales que se caracterizan por una mujer que participa } \\
\text { laboralmente y un hombre inactivo económicamente. }\end{array}$ \\
\hline $\begin{array}{l}\text { Mujer ocupada, hombre } \\
\text { inactivo }\end{array}$ & $\begin{array}{l}\text { Se refiere a las parejas conyugales conformadas por una mujer ocupada y un hombre } \\
\text { inactivo económicamente. }\end{array}$ \\
\hline $\begin{array}{l}\text { Mujer desocupada, hombre } \\
\text { inactivo }\end{array}$ & $\begin{array}{l}\text { Hace referencia a los hogares conyugales conformados por una mujer que participando } \\
\text { en el mercado laboral se encuentra desocupada o en busca de trabajo remunerado y } \\
\text { una hombre inactivo económicamente. }\end{array}$ \\
\hline $\begin{array}{l}\text { TIPO II: } \\
\text { Dos proveedores } \\
\text { Económicos }\end{array}$ & $\begin{array}{l}\text { Son aquellos hogares donde ambos cónyuges participan en el mercado laboral y por } \\
\text { tanto se considera que ambos son proveedores económicos. Para este tipo de pareja se } \\
\text { considera relevante la forma de participación en el mercado laboral, ya sea como } \\
\text { ocupado o desocupado. }\end{array}$ \\
\hline $\begin{array}{l}\text { Hombre ocupado, mujer } \\
\text { ocupada }\end{array}$ & $\begin{array}{l}\text { Se refiere al tipo de hogar conyugal conformado con un hombre y una mujer que se } \\
\text { encuentran ocupados. }\end{array}$ \\
\hline $\begin{array}{l}\text { Hombre ocupado, mujer } \\
\text { desocupada }\end{array}$ & $\begin{array}{l}\text { Abarca a aquellas parejas conyugales que cuentan con un hombre ocupado y una mujer } \\
\text { que participa en el mercado laboral como desocupada. }\end{array}$ \\
\hline $\begin{array}{l}\text { Hombre ocupado, mujer } \\
\text { inactiva con ingresos }\end{array}$ & $\begin{array}{l}\text { Se refiere a las parejas conyugales conformadas por un hombre ocupado y una mujer } \\
\text { inactiva económicamente que cuenta con ingresos por concepto de pensiones, } \\
\text { jubilación, sustitución pensional, invalidez o manutención de menores a cargo de la } \\
\text { pareja conyugal. }\end{array}$ \\
\hline $\begin{array}{l}\text { Hombre desocupado, mujer } \\
\text { ocupada }\end{array}$ & $\begin{array}{l}\text { Son aquellos hogares conyugales conformados con un hombre que se encuentra } \\
\text { desocupado y una mujer ocupada. }\end{array}$ \\
\hline
\end{tabular}

a. La ENUT 2012-2013, considera que participan en el mercado laboral todas aquellas personas disponibles para contribuir a la producción de los bienes y servicios definidos por el Sistema de Cuentas Nacionales de las Naciones Unidas (SCN). Estas personas se clasifican entre ocupadas y desocupadas. La población ocupada corresponde a las personas de 10 años y más, que durante la semana previa a la captura de la información trabajaron al menos 1 hora a cambio de un ingreso monetario o en especie, o trabajaron al menos 1 hora sin recibir pago en calidad de Trabajador Familiar Sin Remuneración o en calidad de Trabajador Sin Remuneración en Empresas de Otros Hogares. También incluye a aquellas personas que no trabajaron (por vacaciones, licencia, etc.), durante el periodo de referencia, pero tenían un empleo o negocio. La población Desocupada corresponde a las personas de 10 años y más que durante la semana pasada estuvieron simultáneamente sin empleo, en busca de empleo o estaban disponibles para empezar a trabajar.

b. En la categoría de inactivo se aborda la condición de estudiando, realizando oficios del hogar, incapacidad permanente o realiza otra actividad, tal como se plantea en el formulario de ENUT 2012 - 2013. 
Los hogares conyugales con doble proveeduría en Colombia. Construcción de una tipología de arreglos laborales con enfoque de género

\begin{tabular}{|c|c|}
\hline $\begin{array}{l}\text { Hombre desocupado, mujer } \\
\text { desocupada }\end{array}$ & $\begin{array}{l}\text { Hace referencia a los hogares conyugales caracterizados por un hombre y una mujer } \\
\text { que se encuentran ambos desocupados. }\end{array}$ \\
\hline $\begin{array}{l}\text { Hombre desocupado, mujer } \\
\text { inactiva con ingresos }\end{array}$ & $\begin{array}{l}\text { Son aquellos hogares conyugales conformados por un hombre que participa del } \\
\text { mercado laboral como desocupado y una mujer inactiva con ingresos por concepto de } \\
\text { pensiones, jubilación, sustitución pensional, invalidez o manutención de menores a } \\
\text { cargo de la pareja conyugal. }\end{array}$ \\
\hline $\begin{array}{l}\text { Hombre inactivo con } \\
\text { ingresos, mujer ocupada }\end{array}$ & $\begin{array}{l}\text { Hace referencia a las parejas conyugales conformadas por un hombre inactivo } \\
\text { económicamente con ingresos por concepto de pensiones, jubilación, sustitución } \\
\text { pensional, invalidez o manutención de menores a cargo de la pareja conyugal y una } \\
\text { mujer que participa laboralmente como ocupada. }\end{array}$ \\
\hline $\begin{array}{c}\text { Hombre inactivo con } \\
\text { ingresos, mujer desocupada }\end{array}$ & $\begin{array}{l}\text { Abarca las parejas conyugales conformadas por un hombre inactivo económicamente } \\
\text { con ingresos por concepto de pensiones, jubilación, sustitución pensional, invalidez o } \\
\text { manutención de menores a cargo de la pareja conyugal y una mujer que participa } \\
\text { laboralmente como desocupada. }\end{array}$ \\
\hline $\begin{array}{l}\text { TIPO III: } \\
\text { Pareja inactiva económica- } \\
\text { mente }\end{array}$ & $\begin{array}{l}\text { Son aquellos hogares conformados por una pareja de cónyuges que se encuentran } \\
\text { inactivos económicamente, puesto que ninguno de estos participa del mercado laboral. }\end{array}$ \\
\hline
\end{tabular}

Fuente: elaboración propia a partir de las categorías analíticas propuestas por Wainerman y Heredia (2000).

Esta tipología permite identificar un conjunto diverso de arreglos laborales de las parejas conyugales que transitan entre: el modelo hegemónico de arreglo familiar de un solo proveedor varón con mujer inactiva laboralmente, sin ningún tipo de participación en el mercado laboral (Tipo IA), así como de una única proveedora mujer con hombre inactivo (Tipo IB). De igual forma, la tipología considera los hogares conyugales de doble proveeduría (Tipo II), como tipo de arreglo laboral emergente cuya configuración se corresponde a factores de orden estructural y coyuntural que, en su conjunto, han presionado a la inserción laboral de las mujeres. En los hogares de doble proveeduría se consideran las opciones simultáneas o alternadas, tanto para hombres como para mujeres, de ocupación y desocupación, dinámicas que dan lugar a una serie de arreglos laborales en las parejas conyugales. En tercer lugar, se consideran las parejas conyugales en las que tanto el hombre como la mujer se encuentran inactivos laboralmente y ninguno de los dos opera como proveedor económico del hogar (Tipo III).

\section{Los hogares con pareja conyugal según número de proveedores, participación laboral y sexo: estimación y clasificación para Colombia}

En este apartado se presenta la clasificación general de los hogares con pareja conyugal en Colombia y se realiza una estimación de la participación de cada uno de los tipos de arreglos laborales, que se han definido en la tipología propuesta. Nos interesa discutir esta morfología de los hogares conyugales en el país, considerando el estatus de la participación en el mercado laboral diferenciada de hombres y mujeres. El análisis se fundamenta en el procesamiento de la información suministrada por la ENUT (2012-2013).

En referencia a la definición operativa utilizada, los hogares con pareja conyugal han sido establecidos como aquellos en los cuales dos personas que pertenecen al mismo hogar declaran tener parentesco correspondiente a jefe o cónyuge y, por tanto, se puede afirmar que conforman una pareja conyugal en ese hogar. Bajo este criterio 
de selección, se consideran sólo los hogares de las áreas urbanas, estimados en 5.767.826 hogares con pareja conyugal, que representan $56,9 \%$ del total de los hogares ubicados en las áreas urbanas del país $^{10}$. Esta alta participación señala la importancia relativa que mantienen los hogares conformados por parejas conyugales en la estructura general de los hogares en Colombia. En tal sentido resulta aún más relevante caracterizar este tipo de hogares y establecer estimaciones con respecto a las diversas combinaciones de arreglos laborales que se pueden encontrar en las parejas conyugales (Tabla 2).

Entre los hogares conformados por parejas conyugales, el tipo de arreglo laboral que predomina es el de un solo proveedor económico, el cual representa 49,8\% del total de los hogares bajo estas

10 En Colombia se estima un total de 13.102.792 hogares. De estos, 10.143.189 se ubican en las áreas urbanas. El análisis realizado considera los hogares de las áreas urbanas y, entre estos, solo aquellos que se definen como hogares de parejas conyugales. Bajo este criterio se excluyen aquellos hogares unipersonales, monoparentales femeninos o masculinos, así como aquellos hogares sin núcleo familiar, entre otros, que representan 43,1\% de los hogares en las áreas urbanas en Colombia. Estas estimaciones se circunscriben a la fuente de datos consultada para la investigación representativa a nivel nacional y a nivel regional, con una muestra compuesta por 148.492 personas y 43.500 hogares a nivel nacional.

Tabla 2. Distribución de los hogares conyugales, según participación laboral por sexo de la pareja conyugal en zonas urbanas, Colombia 2012-2013 (Promedio ponderado) ${ }^{\mathrm{a}}$

\begin{tabular}{|c|c|c|}
\hline Tipo de pareja & Frecuencia absoluta & \% Total hogar nuclear \\
\hline \multicolumn{3}{|l|}{ Un proveedor } \\
\hline Hombre ocupado, mujer inactiva & 2.614 .830 & $45,3 \%$ \\
\hline Hombre desocupado, mujer inactiva & 109.186 & $1,9 \%$ \\
\hline Total un proveedor hombre & 2.724 .016 & $47,2 \%$ \\
\hline Mujer ocupada, hombre inactivo & 145.996 & $2,5 \%$ \\
\hline Mujer desocupada, hombre inactivo & 1.485 & $0,03 \%$ \\
\hline Total una proveedora mujer & 147.481 & $2,6 \%$ \\
\hline Total un proveedor & 2.871.497 & $49,8 \%$ \\
\hline \multicolumn{3}{|l|}{ Dos proveedores } \\
\hline Hombre ocupado, mujer ocupada & 1.860 .239 & $32,3 \%$ \\
\hline Hombre ocupado, mujer desocupada & 31.333 & $0,5 \%$ \\
\hline Hombre ocupado, mujer inactiva con ingresos ${ }^{\mathrm{b}}$ & 104.706 & $1,8 \%$ \\
\hline Hombre desocupado, mujer ocupada & 91.037 & $1,6 \%$ \\
\hline Hombre desocupado, mujer desocupada & 6.259 & $0,1 \%$ \\
\hline Hombre desocupado, mujer inactiva con ingresos & 3.155 & $0,05 \%$ \\
\hline Hombre inactivo con ingresos, mujer ocupada & 54.202 & $0,9 \%$ \\
\hline Hombre inactivo con ingresos, mujer desocupada & 465 & $0,01 \%$ \\
\hline Total dobles proveedores & 2.151 .396 & $37,3 \%$ \\
\hline \multicolumn{3}{|l|}{ Otros } \\
\hline Ambos inactivos (ninguno trabaja) & 744.933 & $12,9 \%$ \\
\hline Total hogar conyugal & 5.767 .826 & $100,0 \%$ \\
\hline Total hogares zonas urbanas & 10.143.189 & \\
\hline
\end{tabular}

a. Como se observa, algunas de las categorías analíticas consideradas en la tipología propuesta no resultan empíricamente significativas -como es el caso de Mujer desocupada-Hombre inactivo o ambos cónyuges desocupados-, sin embargo, se considera relevante incluirlas porque en términos lógicos son tipos posibles y en términos analíticos podrían ser relevantes en circunstancias específicas, cada vez más frecuentes en las sociedades contemporáneas.

b. El término "ingresos" hace referencia a los ingresos del hogar por concepto de pensiones, jubilación, sustitución pensional, invalidez o manutención de menores a cargo de la pareja conyugal, tal como se establece en el formulario de la ENUT (2012-2013).

Fuente: elaboración propia a partir de microdatos de la ENUT (2012-2013), suministrada por el DANE (2012-2013) Colombia. 
Los hogares conyugales con doble proveeduría en Colombia. Construcción de una tipología de arreglos laborales con enfoque de género

características. La mitad de los hogares conformados por parejas conyugales presenta un arreglo laboral donde uno sólo de los cónyuges se encuentra activo laboralmente. No obstante, este alto porcentaje de los hogares con un solo proveedor económico está ampliamente representado por la modalidad de un hombre activo y una mujer inactiva laboralmente, ya que $47,2 \%$ de los hogares se corresponden con este tipo hegemónico y tradicional de arreglo laboral por parte de la pareja conyugal. En tanto, sólo 2,6\% de los hogares conyugales tienen una mujer proveedora económica, donde la mujer es activa y el hombre está inactivo laboralmente. Esta baja participación -del tipo de hogares con una proveedora económica mujer- indica el lugar marginal que aún presentan las mujeres en el arreglo laboral, en los hogares conformados por parejas conyugales (Tabla 2).

Es importante subrayar que el tipo de arreglo laboral de un proveedor hombre también presenta una participación predominante en algunos países de América Latina. En Argentina, se ha reportado que los hogares nucleares completos con hijos con un proveedor hombre ${ }^{\mathrm{Il}}$, aunque han descendido su participación, aún representan más de la mitad del total de estos hogares: en 1991 representaban el 58,1\% y en 1997, el 51,6\% (Wainerman y Heredia, 2000). Así mismo, en México se ha señalado que, del total de parejas conyugales, los hogares con un proveedor hombre representaban $50,7 \%$ en 1992 y redujeron de manera significativa su participación pasando a representar 35,8\%, en el año 2010. Aunque con este valor mantienen su predominio sobre otro tipo de configuraciones (Sánchez, 2014) ${ }^{12}$.

Bajo este marco de referencia, resulta relevante subrayar que los hogares conyugales con doble

11 La información corresponde al área metropolitana de Buenos Aires - Argentina.

12 De acuerdo a la información suministrada para el caso de México, entre 1992 a 2010, se ha presentado el aumento de los hogares con: una solo mujer que trabaja (de 2,1 a 2,4\%), ambos miembros de la pareja que trabajan (de $18,5 \%$ a $24,2 \%$ ), ambos cónyuges que trabajan junto con otro miembro del hogar (de 4,4\% a 10,7\%), y ninguno de los miembros de la pareja trabajan (de $3,1 \%$ a $4,8 \%$ ) (Sánchez, 2014). proveedor representan 37,3\% del total de los hogares conyugales urbanos en Colombia. Estos hogares corresponden al tipo de arreglo laboral donde ambos integrantes se encuentran activos laboralmente u obtienen ingresos bajo modalidades asociadas con su trayectoria laboral. En las estimaciones presentadas se identifica que aquellos hogares donde tanto el hombre como la mujer se encuentran ocupados constituyen la situación más frecuente, equivalente al 32,3\% de los hogares con parejas conyugales urbanas. Para este tipo de arreglo laboral la condición de ocupación de los integrantes de la pareja conyugal define, principalmente, la doble proveeduría. En este contexto, el hogar conyugal con doble proveeduría aparece como el tipo de arreglo laboral emergente que, en principio, prefigura oportunidades de mayor equidad entre hombres y mujeres (Tabla 2).

En términos comparativos, la participación de los hogares conyugales con doble proveedor en Colombia se aproxima a la estimación realizada para otros países en América Latina. Así, tanto en Argentina como en México, la participación de los hogares doble proveedores ha pasado a representar una proporción relevante en las últimas décadas. En el primer caso, de los hogares nucleares completos con hijos, las parejas con ambos cónyuges ocupados pasaron de represen$\operatorname{tar} 30,7 \%$ en 1991 al 31,0\% en 1997 (Wainerman y Heredia, 2000). Y en el caso de México paso de representar el 22,9\% en 1992 al 34,9\% en 2010 (Sánchez, 2014). Sin embargo, en ninguno de estos casos los hogares doble proveedores sobrepasaron los hogares con un proveedor hombre.

Por otro lado, se tienen los hogares conyugales donde ninguno de los integrantes de la pareja participa del mercado laboral, que representan el $12,9 \%$ del total de hogares conyugales urbanos en Colombia. La configuración de este tipo de arreglo laboral conyugal está asociada a un conjunto diverso de situaciones que hacen posible la transferencia de ingresos a estos hogares, entre los cuales se puede señalar: la transferencia de ingresos de otros miembros de la familia a la pareja conyugal (provenientes de fuera y/o dentro del país); ingresos por rentas de propiedades de la pareja conyugal; ingresos por pensiones, 
jubilación, sustitución pensional, invalidez o manutención de menores a cargo de la pareja conyugal (Tabla 2) ${ }^{13}$.

A partir de la clasificación de los hogares conformados por parejas y la estimación de su magnitud según el tipo de arreglos laborales presentada para las áreas urbanas en Colombia, hemos constatado la importancia que adquiere la configuración emergente de los hogares con doble proveeduría en el país. Este conjunto de hogares resulta altamente significativo para comprender procesos de cambio social, en una perspectiva mucho más amplia, que están fuertemente asociados con dinámicas de transformación en las estructuras demográficas, en la participación de las mujeres en el mercado laboral y en los roles de hombres y mujeres en la dimensión de la vida pública; así como en las dinámicas de la vida privada y las relaciones de pareja fuertemente vinculadas con la división sexual del trabajo doméstico y de cuidado.

\section{Los hogares conyugales de doble proveeduría en Colombia: un tipo predominante en las parejas con altos niveles educativos}

Las investigaciones realizadas en diversos países de Latinoamérica, que han centrado su in-

13 De acuerdo a la información procesada, el 70,2\% de los hogares conyugales con ambos miembros de la pareja inactivos, logran el sostenimiento económico del hogar mediante los ingresos de, mínimo, uno de los cónyuges por concepto de: primero, pensiones, jubilación, sustitución pensional, invalidez o manutención de menores a cargo de la pareja conyugal; segundo, la transferencia de ingresos de otros miembros de la familia a la pareja conyugal, provenientes de fuera y/o dentro del país; y tercero, rentas de propiedades de la pareja conyugal. Con mayor frecuencia, el sostenimiento de estos hogares proviene de: solo pensiones y afines $(40,8 \%)$, transferencias de ingresos de otros hogares (12,8\%), y rentas (5,5\%). Así mismo, se presentan otros tipos de arreglos más complejos. Estos se caracterizan por hogares que se sostienen económicamente a partir de dos tipos de ingresos. Se refiere a: pensiones y rentas $(6,8 \%)$, pensiones y transferencias de ingresos de otros hogares $(2,2 \%)$, y rentas y transferencias de ingresos de otros hogares $(2,0 \%)$. terés en los hogares con doble proveeduría, han auscultado diversas dimensiones con el propósito de caracterizar este tipo de hogar conyugal y aportar a la comprensión de su mayor participación en la estructura general de los tipos de hogares, en las sociedades contemporáneas. En tal sentido, estudios como los de Wainerman y Heredía (2000), que indagan el caso particular del área metropolitana de Buenos Aires, señalan que en contextos de crisis económica -como el experimentado en Argentina en las décadas de los ochenta y noventa- resulta mucho más crítico el sostenimiento de los hogares con un único ingreso. Esto explica el crecimiento de los hogares doble proveedores en diferentes sectores sociales y en distintos momentos del ciclo de vida familiar.

Este crecimiento de las parejas de doble proveeduría, dentro de la estructura de hogares de Buenos Aires, presenta una participación de forma diferenciada según las condiciones socioeconómicas. Así que aquellos hogares de altas condiciones económicas son los que de forma más rápida y temprana han adoptado el modelo de doble proveedores y han contado con una mayor concentración de estas parejas (Wainerman y Heredia, 2000). Este crecimiento obedece no solo a la falta de recursos económicos -dado en sectores de bajos ingresos, en mayor medidasino también a los altos niveles de educación ${ }^{14}$ en sectores de mejor situación económica. De tal manera que los mayores niveles de educación se presentan como un factor relevante, puesto que brindan una mayor oportunidad de inserción laboral a la población femenina, así como influyen en cambios culturales con el fomento de valores que conllevan al desarrollo de una vida profesional, tanto en mujeres como en hombres.

Por otro lado, Landy Sánchez (2014) señala en su estudio sobre las parejas de doble ingreso en México, primero, que estos hogares dan cuentan del aumento en la participación laboral femeni-

14 De acuerdo a la investigación de Wainerman y Heredia (2000), los niveles de educación altos incluyen la educación secundaria completa, así como las categorías de superior y posgrado. 
Los hogares conyugales con doble proveeduría en Colombia. Construcción de una tipología de arreglos laborales con enfoque de género

na, y de quienes se incorporan y permanecen en el mercado laboral. Y, en segundo lugar, la autora hace énfasis en la concentración de este tipo de parejas en los "estratos de ingresos más altos, reflejando tanto tendencias de homogamia marital como su mayor capacidad económica al contar con dos perceptores" (p. 471); sugiriéndose, de esta manera, que los hogares de doble proveeduría responden a un criterio de estratificación económica y selectividad social.

Un conjunto de investigaciones realizadas en diversos contextos coincide en señalar que las parejas de doble ingreso son las que presentan un reparto más igualitario o simétrico en el uso del tiempo (Batthyáni, 2015; García, 2012). Sin embargo, también enfatizan en la persistencia de brechas de género en el trabajo doméstico y de cuidado en este tipo de hogares (Puyana y Mosquera, 2003; Sosa, 2015). En general, estas investigaciones señalan que, incluso en los hogares de doble proveeduría, las actividades domésticas siguen considerándose de menor valor social y siguen siendo asignadas, principalmente, a las mujeres, estableciéndose de esta manera condiciones de desventaja social, fundamentadas en el género (Batthyáni, 2015; Santoyo y Pacheco, 2014; Wainerman y Heredia, 2000).

Entre los hallazgos de las investigaciones recientes, dos aspectos cobran importancia y resultan relevantes a subrayar. Por un lado, en los hogares de doble proveeduría es relativamente frecuente que se acuda a la contratación de trabajo doméstico externo, como una estrategia para compensar el uso del tiempo dedicado por la pareja a estas actividades y, aunque en la mayoría de los casos se contrata trabajo femenino, esta alternativa constituye una opción de mayor equidad, principalmente para las mujeres (García, 2012; Monroy y Olarte, 2015; Puyana y Mosquera, 2003). No obstante, esta estrategia está circunscrita a las condiciones económicas de los hogares y resulta factible para hogares de altos ingresos (Puyana y Mosquera, 2003; Sánchez, 2014).

De otro lado, se ha identificado que las parejas de doble ingreso que presentan características distanciadas del modelo tradicional de familia -cohabitar en unión libre, percibir ingresos simi- lares, no tener hijos y contar con nivel educativo universitario- tienen un reparto más igualitario y simétrico del tiempo (García, 2012). Estos hallazgos ponen de presente la importancia que adquieren diversos capitales y factores de poder, incluso en la configuración de los hogares de doble proveeduría.

En el contexto de los hallazgos presentados nos interesa centrar la atención en la importancia que adquieren los niveles educativos en los hogares de doble proveeduría. En la misma dirección del planteamiento señalado por García (2012) y Wainerman y Heredia (2000), consideramos que los niveles educativos, en los hogares doble proveedores, son un factor fundamental en el análisis de la configuración de este tipo de hogares, tal como se describe a continuación para el caso de las zonas urbanas de Colombia.

El análisis detallado de los niveles educativos de los hogares doble proveedores en Colombia señala una clara asociación entre el tipo de arreglo laboral de la pareja conyugal y el nivel educativo alcanzado por la pareja. Mientras que los cónyuges de hogares con un proveedor hombre presentan una concentración significativa en el nivel educativo de primaria y menos (52,0\%), y en el nivel de secundaria o media (54,2\%), los hogares con doble proveedor cuentan con una participación relevante en el nivel educativo superior $(50,1 \%)$ y en educación de posgrado (60,2\%) (Gráfico 1$)^{15}$.

Las parejas conyugales en Colombia, con un tipo de arreglo laboral de carácter tradicional, presentan una mayor concentración en los hogares con menor nivel educativo. En contraste, se observa que otros tipos de parejas conyugales, con una organización familiar más igualitaria en los roles económicos de cada cónyuge, como el hogar con doble proveedor, se ubica en mayor medida en hogares con niveles educativos más altos. En tal sentido, se puede señalar una asociación entre el nivel educativo alto y el tipo de arreglo de doble proveedor en un hogar conyugal.

15 Se estimó la asociación entre la variable tipo de hogar y nivel educativo mediante la prueba del chi cuadrado. Los resultados obtenidos permiten señalar la asociación entre estas variables. 
Gráfica 1. Participación en el nivel educativo de los tipos de hogares conyugales según arreglo laboral en zonas urbanas, Colombia 2012-2013 (en porcentajes)

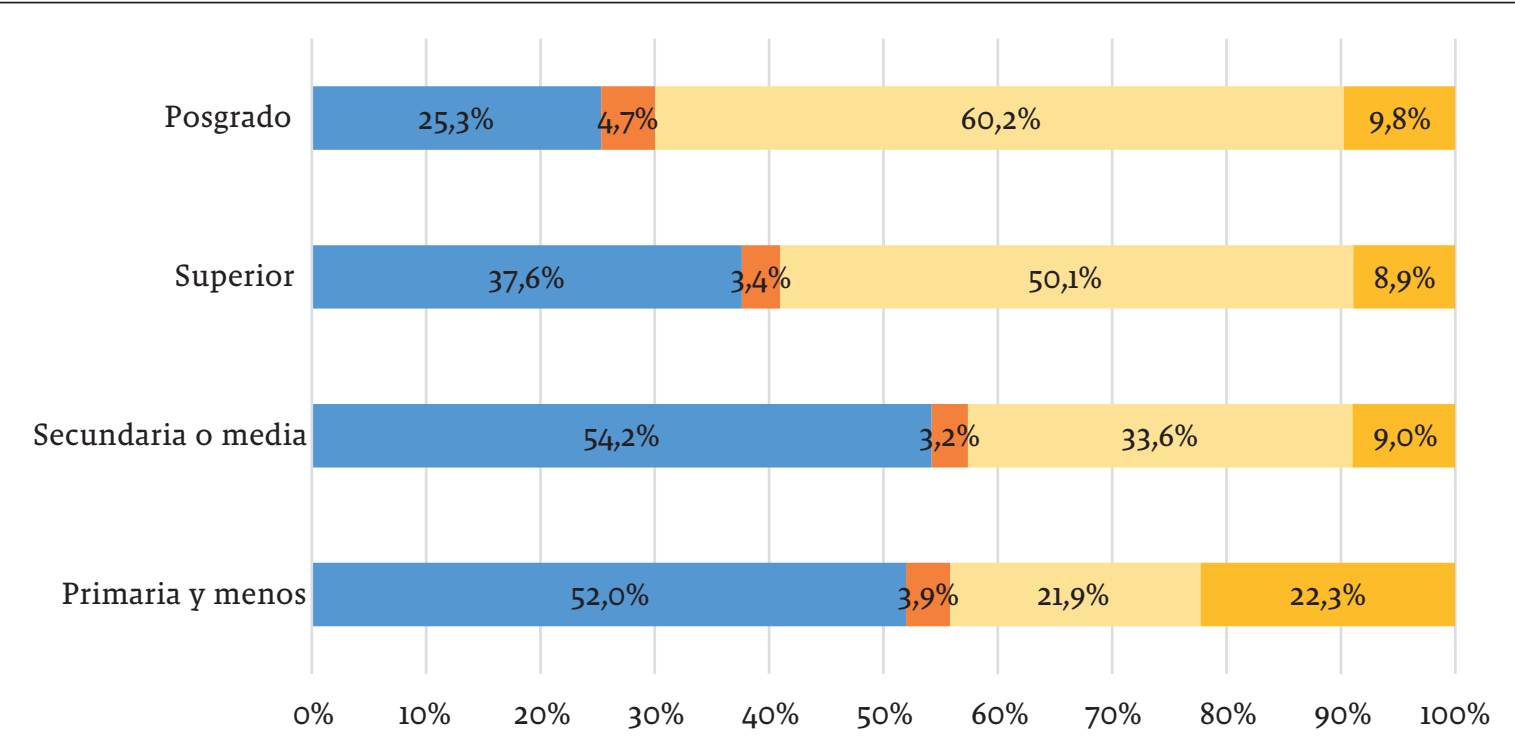

- Un Proveedor Hombre Una proveedora Mujer Doble proveedores Ambos inactivos

Fuente: elaboración propia a partir de microdatos de la ENUT (2012-2013), suministrada por el DANE, Colombia.

De igual forma, se observa que los hogares con ambos cónyuges inactivos se ubican principalmente en el nivel de primaria y menos $(22,3 \%)$, reduciendo su participación en el resto de niveles educativos (alrededor del 9\%). En tal sentido, en estos hogares se puede señalar una asociación con bajos niveles educativos, debido a una alta participación de población con rangos de edad de 55 años y más ${ }^{16}$. Población que tiende a presentar un promedio de años de estudio considerablemente bajo (Guzman y Sosa, 2002).

En igual dirección, en los hogares con una proveedora mujer -aunque cuenten con una escasa participación en cada uno de los niveles educativos- se observa de forma particular que la concentración de estas parejas es relativamente mayor en el nivel de educación de primaria $y$ menos (3,9\%) y de posgrado ( $4,7 \%)$, ubicándose en

16 De acuerdo a la información suministrada por la ENUT (2012-2013), el 76,3\% de los cónyuges que conforman los hogares con ambos inactivos presentan edades de $55 \mathrm{y}$ más años. De esta población adulta mayor, $46,9 \%$ se ubica en el nivel educativo de primaria y menos. posiciones opuestas en la estratificación educativa. Lo cual puede relacionarse con la forma de inserción y permanencia en el mercado laboral por parte de las mujeres con estos niveles educativos: tanto por la falta de recursos económicos de sus hogares, por un lado, como por los cambios culturales relacionados a un mayor capital educativo, por otro, que tiende a afectar la organización de los roles económicos de estas parejas en los dos extremos educativos, respectivamente (Gráfico 1).

En tal sentido, se puede afirmar que las parejas con niveles educativos más bajos están asociadas con hogares con arreglos laborales de un solo proveedor, en tanto que las parejas con altos niveles educativos están asociadas con arreglos laborales de doble proveeduría (Gráfico l).

El importante peso que tienen los altos niveles educativos en los hogares doble proveedores se hace mucho más explícito al indicar que, tanto para las mujeres como para los hombres, se concentra la mayor participación en el nivel educativo superior ( $32,6 \%$ y $25,8 \%$, respectivamente) 
Los hogares conyugales con doble proveeduría en Colombia. Construcción de una tipología de arreglos laborales con enfoque de género

y de posgrado $(6,3 \%$ y $5,8 \%)$. En esta misma dirección, en los hogares doble proveedores se identifican las participaciones más bajas en los menores niveles educativos en ambos sexos. Esta clara asociación entre mayor nivel educativo y hogares doble proveedores se hace mucho más importante al considerar la mayor participación de las mujeres en los altos niveles educativos, que supera en términos relativos la participación de los hombres. De forma complementaria, se subraya que son las mujeres de los hogares con doble proveeduría las que presentan menor participación en el nivel de educación primaria y menos $(17,5 \%)$, ratificándose así que los hogares con doble proveeduría se apuntalan en un alto capital escolar de las parejas y, principalmente, de las mujeres (Gráfica 2).

La estrecha relación entre alto nivel educativo y configuración de hogares de doble proveeduría se ilustra de mejor manera al considerar los hogares conyugales conformados por hombres y mujeres pertenecientes a las cohortes que se ubican en el rango de edad entre 25 y 40 años, por tratarse de una población más selectiva: más joven, en edad productiva y más escolarizada. Los hogares con doble proveeduría participan de manera más significativa en la población con más alto nivel educativo: entre la población que ha realizado estudios de posgrados los hogares con doble proveedores representan $74,1 \%$, en tanto que entre la población con nivel educativo de primaria y menos, los hogares con doble proveedor solo representan 32,5\% (Gráfica 3).

Este conjunto de estimaciones, en torno a los hogares conyugales de doble proveedores, permite subrayar no solo la creciente participación de este tipo de hogares, sino también enfatizar en la estrecha relación con la población con altos niveles educativos. Dicho hallazgo afirma que, la configuración de este tipo de hogares, constituye una importante apertura en el establecimiento de dinámicas más equitativas en los ámbitos familiares, asociadas con cambios culturales en las relaciones más democráticas que se configuran en los espacios privados.

Gráfica 2. Distribución de los tipos de hogares conyugales por arreglo laboral, según sexo y nivel educativo, en zonas urbanas de Colombia, 2012-2013 (en porcentajes)

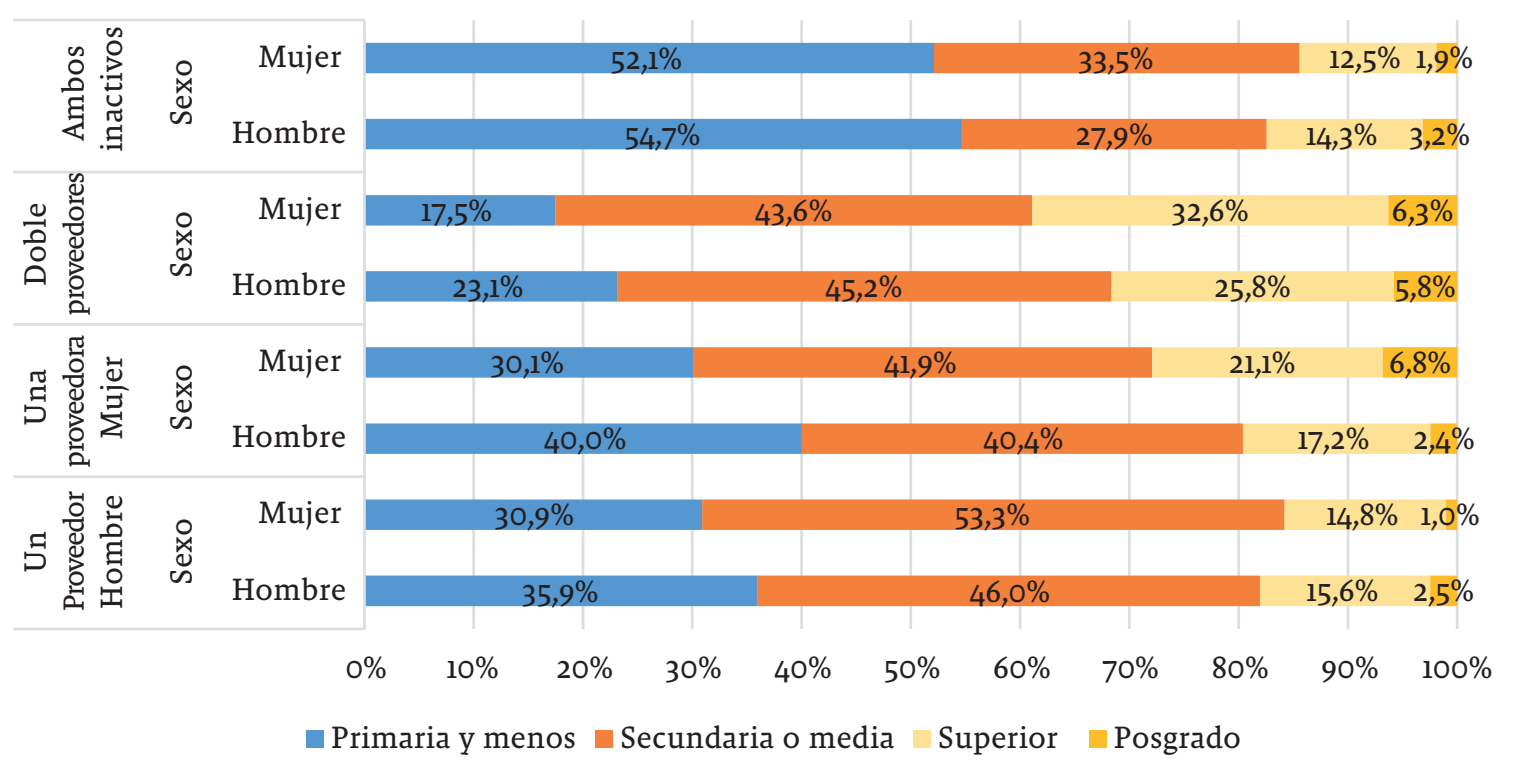

Fuente: elaboración propia a partir de microdatos de la ENUT (2012-2013), suministrada por el DANE, Colombia. 
Gráfica 3. Participación de la población entre 25 y 40 años de edad en el nivel educativo, según los tipos de hogares conyugales y arreglo laboral en zonas urbanas, Colombia 2012-2013 (en porcentajes)

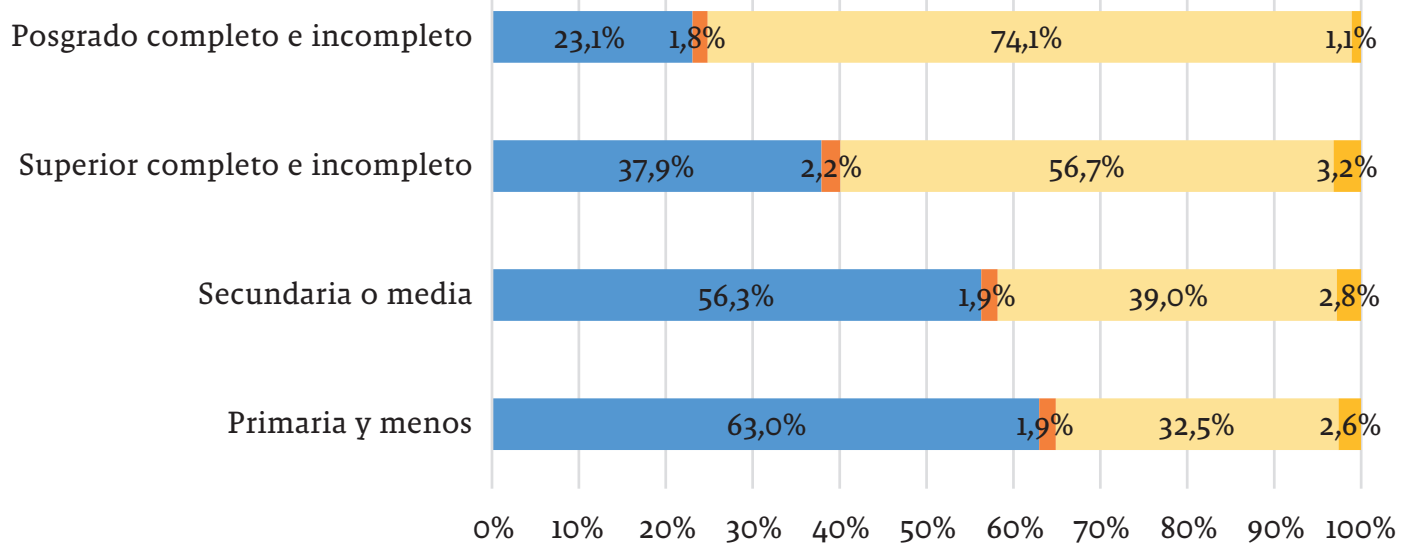

- Un proveedor hombre una proveedora mujer Doble proveedores Ambos inactivos

Fuente: elaboración propia a partir de microdatos de la ENUT (2012-2013), suministrada por el DANE, Colombia.

\section{Reflexiones finales}

El análisis que hemos presentado en torno a la estructura general de los hogares en Colombia, se instala en la discusión sobre el proceso de transición del hogar tipo con un único proveedor económico hombre, hacia la emergencia de una diversidad de tipos de hogares, que han puesto de presente la masiva participación en el mercado laboral de las mujeres y su rol como proveedoras económicas. En el contexto general de este debate presentamos una propuesta de tipología de los hogares conyugales, que conjuga la participación en el mercado laboral de hombres y de mujeres, y el número de proveedores económicos del hogar según el sexo. Esta tipología permite distinguir tres grandes tipos de hogares: hogar con único proveedor, hogar con doble proveeduría y hogar sin proveedor económico. En cada uno de estos tres grandes tipos se configuran distintas combinaciones que diferencian el estatus laboral de cada uno de los cónyuges, dando cuenta del conjunto de posibilidades de arreglos laborales que se establecen en los hogares conyugales en la actualidad.
El análisis realizado de la estructura de hogares en Colombia -utilizando la tipología propuesta y procesando la información suministrada por la Encuesta Nacional de Uso del Tiempo (ENUT 2012-2013)- ha permitido estimar que los hogares con doble proveeduría, en Colombia, representan $37,3 \%$ del total de los hogares conyugales urbanos en el país. De esta forma, se constata la transición hacia este tipo de hogares que dan cuenta de una mayor participación laboral de las mujeres y de su rol como proveedoras económicas en los hogares conyugales. En el marco de los hogares doble proveedores, se identifican un conjunto de arreglos laborales específicos, que dan cuenta de una amplia diversidad en este tipo de hogares; sin embargo, el peso de aquellos en los cuales ambos cónyuges se encuentran ocupados resulta predominante, representado $32,3 \%$.

En la configuración de los hogares de doble proveeduría, resulta relevante subrayar dos aspectos que aparecen claramente asociados al aumento que estos hogares presentan en la actualidad. De un lado, la permanencia de las mujeres en el mercado laboral se constituye en 
Los hogares conyugales con doble proveeduría en Colombia. Construcción de una tipología de arreglos laborales con enfoque de género

un factor determinante en la transformación de los roles de género y en la representación de las mujeres como proveedoras económicas en la sociedad contemporánea. De otro lado, se identifica que la configuración de los hogares con doble proveeduría, gana mayor predominio entre parejas con altos niveles educativos: entre los hogares con nivel educativo superior, aquellos con doble proveeduría representan 50,1\% y, entre las parejas conyugales con nivel educativo de posgrado, los hogares doble proveedores representan $60,2 \%$.

Los rasgos señalados que caracterizan las parejas con doble proveeduría, es decir, la mayor participación laboral de las mujeres y el mayor nivel educativo de los cónyuges -principalmente de las mujeres- permiten sugerir que, en este tipo de hogares, hay mayor probabilidad de que se establezcan condiciones que favorezcan la igualdad de género. Así mismo, tendrían mejores condiciones para que se adopten relaciones y prácticas en la distribución del trabajo doméstico y de cuidado, que expresen redefiniciones de poder en la pareja, asociadas con este cambio de roles. Estos hallazgos se instalan en los debates centrales de los estudios del trabajo desde la perspectiva de género y reeditan la discusión en torno a categorías analíticas como la división sexual del trabajo, enfatizando en el análisis de las dinámicas internas en estos hogares. De tal manera que se plantea un amplio debate en torno a la importancia de identificar en qué medida estos nuevos arreglos laborales, en los hogares conyugales, están asociados a la redefinición del mundo laboral y en qué medida han significado cambios en el mundo privado (García y de Oliveira, 2006).

El análisis desarrollado no alcanza a dilucidar en qué medida los procesos de cambio cultural se están desarrollando en la sociedad colombiana, pero sí nos permite señalar la importancia que ha adquirido realizar investigaciones en esta dirección. Solo de esta manera se podrá dar cuenta del grado de avance en materia de equidad de género en las familias, que hoy en día representa uno de los grandes desafíos, no solo en la esfera privada sino como problemática de carácter público, que incumbe tanto al Estado como al mercado.

\section{Referencias}

Ariza, M. y de Oliveira, O. (2009). Desigualdades sociales y relaciones intrafamiliares en el México del siglo XXI. En C. Rabell, Tramas familiares en el México contemporáneo. Una perspectiva sociodemográfica (pp. 257-291). Ciudad de México, México: Instituto de Investigaciones Sociales, El Colegio de México.

Ariza, M. y de Oliveira, O. (2001). Familias en transición y marcos teóricos en redefinición. Papeles de Población, (28), 9-39.

Arriagada, I. (2004). Cambio de las familias en el marco de las transformaciones globales: Necesidad de políticas públicas eficaces. Santiago, Chile: CEPAL, Naciones Unidas.

Arriagada, I. y Aranda, V. (2004). Cambio de las familias en el marco de las transformaciones globales: necesidades de políticas públicas eficaces. Santiago, Chile: CEPAL.

Batthyáni, K. (2015). Cuando las mujeres son breadwinners ¿quién asume el trabajo no remunerado? En N. Genta y V. Perrota, Los tiempos del bienestar social. Género, trabajo no remunerado y cuidados en Uruguay (pp. 135-170). Montevideo, Uruguay: INMUJERES - MIDES.

DANE -Departamento Administrativo Nacional de Estadística-. Encuesta Nacional del Uso del Tiempo, 2012-2013. Recuperado de https:/www.dane.gov.co/index.php/estadisticas-por-tema/ pobreza-y-condiciones-de-vida/encuesta-nacional-del-uso-del-tiempo-enut

García, B. y de Oliveira, O. (2006). La familia y el trabajo: principales enfoques teóricos e investigaciones sociodemográficas. En E. de la Garza (Coord.), Tratado Latinoamericano de Sociología (pp. 148-170). 
Barcelona/Ciudad de México, España/México: Anthropodos Editorial, Universidad Autónoma Metropolitana -Iztapalapa.

García, J. (2012). El uso del tiempo en las parejas de doble ingreso (tesis doctoral). Universidad Autónoma de Barcelona, Barcelona, España.

Guzmán, J. M. y Sosa, Z. (2002). Los adultos mayores en América Latina y el Caribe. Datos e indicadores. Santiago, Chile: CEPAL.

Jelin, E. (2007). Las familias latinoamericanas en el marco de las transformaciones globales. En I. Arriagada, Familias y políticas públicas en América Latina: Una historia de desencuentros (pp. 7792). Santiago, Chile: CEPAL.

Martínez, C. (2013). Descenso de la fecundidad, participación laboral de la mujer y reducción de la pobreza en Colombia, 1990-2010. Bogotá, Colombia: Profamilia.

Ministerio de Salud y Protección Social y Profamilia. (2016). Resumen Ejecutivo. Encuesta Nacional de Demografía y Salud ENDS Colombia 2015. Recuperado de https://dhsprogram.com/pubs/pdf/ FR334/FR334.pdf

Monroy, V. y Olarte, M. A. (2015). Estudio sobre el comportamiento de la división del trabajo en el hogar: particularidades de género para Colombia. En Fondo De Población De Las Naciones Unidas - UNFPA; DANE, Investigas: Siete estudios realizados a partir de la Encuesta Nacional del Uso del Tiempo, 2012-2013 (pp. 118-141). Bogotá, Colombia: Fondo de Población de las Naciones Unidas.

Puyana, Y. y Mosquera, C. (2003). El trabajo doméstico y la proveeduría en la ciudad de Bogotá: Cambios y persistencias. En Y. Puyana, Padres y madres en cinco ciudades: cambios y permanencias (pp. 149-187). Bogotá, Colombia: Universidad Autónoma de Bucaramanga, Universidad del Valle, Universidad de Cartagena, Universidad de Antioquia y Universidad Nacional de Colombia.

Quilodrán, J. (2011). La familia referentes en transición. En J. Quilodrán, Parejas conyugales en transformación (pp. 53-98). Ciudad de México, México: El Colegio de México.

Rendón, T. (2004). El mercado laboral y la división intrafamiliar del trabajo. En M. Ariza y O. de Oliveira, Imágenes de la familia en el cambio de siglo (pp. 49-87). Ciudad de México, México: Universidad Autónoma de México.

Rodríguez, C. (2015). Economía feminista y economía de cuidado. Aportes conceptuales para el estudio de la desigualdad. Revista Nueva Sociedad, (256), 30-44.

Sánchez, L. (2014). Desigualdad y trabajo doméstico en las parejas de doble ingreso en México. En B. García y E. Pacheco, Uso del tiempo y el trabajo no remunerado en México (pp. 471-507). Ciudad de México, México: El Colegio de México, Centro de Estudios Demográficos.

Santoyo, L. y Pacheco, E. (2014). Uso del tiempo de las personas en México según tipo de hogar. Una expresión de las desigualdades de género. En B. García y E. Pacheco, Uso del tiempo y trabajo no remunerado en México (pp. 171-219). Ciudad de México, México: El Colegio de México, ONU Mujeres, Instituto Nacional de las Mujeres.

Sosa, M. V. (2015). Participación y tiempo en actividades cotidianas de hombres y mujeres vinculados al mercado laboral en México. Sociedad y Economía, (29), 63-89. 
Los hogares conyugales con doble proveeduría en Colombia. Construcción de una tipología de arreglos laborales con enfoque de género

Therborn, G. (2007). Familias en el mundo. Historia y futuro en el umbral del siglo XXI. En I. Arriagada (coord.), Familia y políticas públicas en América Latina: una historia de desencuentros (pp. 31-61). Santiago, Chile: Naciones Unidas.

Ullmann, H., Maldonado, C. y Nieves, M. (2014). La evolución de las estructuras familiares en América Latina, 1990-2010. Los retos de la pobreza, la vulnerabilidad y el cuidado. Santiago, Chile: Naciones Unidas.

Wainerman, C. (2007). Conyugalidad y paternidad ¿Una revolución estancada? En M. A. Gutiérrez, Desafíos para la investigación política (pp. 149-185). Buenos Aires, Argentina: CLACSO, Consejo Latinoamericano de Ciencias Sociales.

Wainerman, C. y Heredia, M. (2000). El trabajo en familia de dobles proveedores: producción y reproducción. Buenos Aires, Argentina: III Congreso Latinoamericano de Sociología del Trabajo.

West, C. y Zimmerman, D. (1999). Haciendo género. En M. Navarro y C. R. Stimpson (coords.), Sexualidad, género y roles sexuales (pp. 109-143). Buenos Aires, Argentina: Fondo de Cultura Económica. 